\title{
COVID-19 in children with underlying chronic respiratory diseases: survey results from 174 centres
}

\author{
Alexander Moeller (10)11, Leo Thanikkel ${ }^{1,11}$, Liesbeth Duijts (10), \\ Erol A. Gaillard ${ }^{4,5}$, Luis Garcia-Marcos ${ }^{6}$, Ahmad Kantar (10) Nathalie Tabin ${ }^{8}$, \\ Steven Turner $\mathbb{1}^{9}$, Angela Zacharasiewicz ${ }^{10}$ and Mariëlle W.H. Pijnenburg ${ }^{2}$
}

\section{ABSTRACT}

Background: Early reports suggest that most children infected with severe acute respiratory syndrome coronavirus 2 ("SARS-CoV-2") have mild symptoms. What is not known is whether children with chronic respiratory illnesses have exacerbations associated with SARS-CoV-2 virus.

Methods: An expert panel created a survey, which was circulated twice (in April and May 2020) to members of the Paediatric Assembly of the European Respiratory Society (ERS) and via the social media of the ERS. The survey stratified patients by the following conditions: asthma, cystic fibrosis (CF), bronchopulmonary dysplasia (BPD) and other respiratory conditions.

Results: In total 174 centres responded to at least one survey. 80 centres reported no cases, whereas 94 entered data from 945 children with coronavirus disease 2019 (COVID-19). SARS-CoV-2 was isolated from 49 children with asthma of whom 29 required no treatment, 19 needed supplemental oxygen and four children required mechanical ventilation. Of the 14 children with CF and COVID-19, 10 required no treatment and four had only minor symptoms. Among the nine children with BPD and COVID-19, two required no treatment, five required inpatient care and oxygen and two were admitted to a paediatric intensive care unit (PICU) requiring invasive ventilation. Data were available from 33 children with other conditions and SARS-CoV-2 of whom 20 required supplemental oxygen and 11 needed noninvasive or invasive ventilation.

Conclusions: Within the participating centres, in children with asthma and CF, infection with SARS-CoV2 was well tolerated, but a substantial minority of children with BPD and other conditions required ventilatory support indicating that these latter groups are at risk from SARS-CoV-2 infection.

$@$ ERSpublications

Whereas children with asthma and CF seem not to be at increased risk of severe \#COVID19, a minority of children with other respiratory diseases might be at increased risk and may benefit from being shielded https://bit.ly/3fiwUXp

Cite this article as: Moeller A, Thanikkel L, Duijts L, et al. COVID-19 in children with underlying chronic respiratory diseases: survey results from 174 centres. ERJ Open Res 2020; 6: 00409-2020 [https://doi.org/10.1183/23120541.00409-2020].

This article has supplementary material available from openres.ersjournals.com

Received: 22 June 2020 | Accepted after revision: 27 July 2020

Copyright $\odot$ ERS 2020. This article is open access and distributed under the terms of the Creative Commons Attribution Non-Commercial Licence 4.0. 


\section{Introduction}

The novel severe acute respiratory syndrome coronavirus 2 (SARS-CoV-2) has spread rapidly from Wuhan, China around the world within 100 days [1]. Western industrialised nations have been hotspots for coronavirus disease 19 (COVID-19), with the USA, UK, Italy, Spain, France, Russia and Belgium severely affected and overtaking China's death toll. Many health systems have struggled to cope with a sudden surge of COVID-19 patients and have been forced to scale up their operations to meet this demand.

Children have been relatively spared from severe COVID-19-related illness and represented only $1.2 \%$ of the caseload in Italy [2], 2\% in China [3] and 5\% in the USA [4]. Where children are infected, they are usually asymptomatic, or have mild symptoms, and do not require hospitalisation [5]. One potential severe complication of SARS-CoV-2 infection in children is a new disease variant that has been noted through the use of early surveillance tools, with the possible emergence of a Kawasaki-like disease [6]. The apparently low burden of morbidity in children from COVID-19 has made the impact of infection on children difficult to ascertain. Reviews, such as those carried out by the Chinese Center for Disease Control and Prevention, describe the clinical features, epidemiological characteristics and radiological findings of fewer than 200 children [7]. SARS-CoV-2 is a respiratory virus, and what remains unknown is whether children with chronic respiratory conditions are at increased risk from SARS-CoV-2-driven exacerbations. As a precaution against SARS-CoV-2 infection causing problems in children with asthma, the UK government advised that children on additional preventer treatment should remain shielded (including remaining inside the home at all times and keeping $2 \mathrm{~m}$ away from household contacts indoors).

Here we report the results of a survey sent to members of the Paediatric Assembly of the European Respiratory Society (ERS) and published via the social media of the ERS covering centres within and outside of Europe that was designed to determine the number of cases of children with pre-existing chronic respiratory conditions who presented to hospital and had SARS-CoV-2 identified and were therefore classified as COVID-19 cases. The survey also captured characteristics and management of cases.

\section{Methods}

Survey design

In March 2020 an expert group of members from the ERS developed a survey to collect basic information on children who tested positive for SARS-CoV-2 during the pandemic. The survey primarily sought to determine the number of cases. Additionally, the survey captured characteristics of cases, their management and in a limited number the outcome after 2 weeks. We conducted a survey only, and the data collected was fully anonymised. Ethics approval was not thought necessary.

The first survey (see details in supplementary table S1) was structured into two sections: 1) basic questions about the number of children who tested positive at the institution including age, sex, underlying respiratory conditions, hospitalisation, paediatric intensive care unit (PICU) treatment and death; and 2) specific questions about children with asthma, cystic fibrosis (CF), bronchopulmonary dysplasia (BPD) and other respiratory or general health conditions. These included the main clinical presentation (i.e. exacerbation, pneumonia, upper respiratory tract infection, fever only, asymptomatic or others), admission to a children's ward or PICU, length of hospitalisation and treatment (supplemental oxygen, noninvasive ventilation (NIV) or invasive ventilation). Data was also collected on other medical treatments such as antibiotics and experimental COVID-19 drugs.

The follow-up survey consisted of two parts. The first part contained questions regarding clinical follow-up of children reported in the first round of the survey 3 weeks earlier (details in supplementary

Affiliations: 'Division of Respiratory Medicine and Childhood Research Center, University Children's Hospital Zurich, Zurich, Switzerland. ${ }^{2}$ Dept of Pediatrics, Division of Respiratory Medicine and Allergology, Erasmus MC, University Medical Center Rotterdam, Rotterdam, The Netherlands. ${ }^{3}$ Dept of Pediatrics, Division of Neonatology, Erasmus MC, University Medical Center Rotterdam, Rotterdam, The Netherlands. ${ }^{4}$ Dept of Respiratory Sciences, College of Life Sciences, University of Leicester, Leicester, UK. ${ }^{5}$ NIHR Biomedical Research Centre (Respiratory Theme), Leicester, UK. ${ }^{6}$ Pulmonology and Allergy Units, Arrixaca University Children's Hospital, University of Murcia, Biohealth Research Institute of Murcia (IMIB), and ARADyAL network, El Palmar, Spain. ${ }^{7}$ Pediatric Asthma and Cough Center, Istituti Ospedalieri Bergamaschi, Bergamo, Ponte San Pietro, Italy. ${ }^{8}$ European Respiratory Society, Lausanne, Switzerland. ${ }^{9}$ Child Health, University of Aberdeen, Aberdeen, UK. ${ }^{10}$ Dept of Pediatrics, Wilhelminenspital, Klinikum Ottakring, Teaching Hospital of the University of Vienna, Vienna, Austria. ${ }^{11}$ These authors contributed equally.

Correspondence: Alexander Moeller, Division of Respiratory Medicine, University Children's Hospital Zürich, Steinwiesstrasse 75, 8032 Zürich, Switzerland. E-mail: alexander.moellerlakispi.uzh.ch 
table S2). The second part asked for information about new cases and contained the same questions as the first survey.

\section{Survey dissemination}

The survey, generated using SurveyMonkey, was sent out from the ERS office via the "officer tool" by e-mail to all members of the ERS Paediatric Assembly. The survey was also promoted via social media, including on the official ERS accounts for Twitter and Facebook. The first survey was launched on 30 March 2020, ending on 8 April 2020, and the follow-up survey was sent out on 18 April 2020, ending on 3 May 2020.

\section{Analysis}

Data were checked for multiple entries from the same institutions, and inconsistent or invalid data. Where necessary, physicians were contacted by e-mail to request clarification. Occasionally two individuals from the same hospital returned a report with different data. This was the case in some hospitals with distinct clinics, such as a CF centre or an asthma clinic separate from the paediatric respiratory clinic. In such cases the entered data was checked individually to exclude double reporting.

\section{Results}

\section{Survey results}

The survey was sent to 1452 members of the ERS Paediatric Assembly in 456 centres. In addition, 49 non-members opened the survey via the social media platforms. A total of 274 individuals from 106 centres in 35 countries responded to the first survey (supplementary table S3). From the 274 respondents, only $122(44.5 \%)$ from 107 centres in 43 countries entered more data than their name and institution. Fourteen centres entered data twice, and in four of these, individuals reported data from different clinics, resulting in 112 datasets for analysis. The second survey yielded responses from 110 physicians from 101 centres in 33 countries. Twenty-two of the responses were entered by non-members of the ERS Pediatric Assembly. Elimination of six double-entries resulted in a total of 104 datasets. From those individuals responding to the second survey, 62 stated that they had not provided answers to the first survey (figure 1).

Of the 112 centres responding to the first survey, 52 reported no paediatric COVID-19 cases, while 60 entered data from at least one child who tested positive for SARS-CoV-2. Of the 110 respondents of the second survey, 28 stated that they had not seen children who tested positive for SARS-CoV-2 until 8 April

First survey

30 March 2020, ending on 8 April 2020

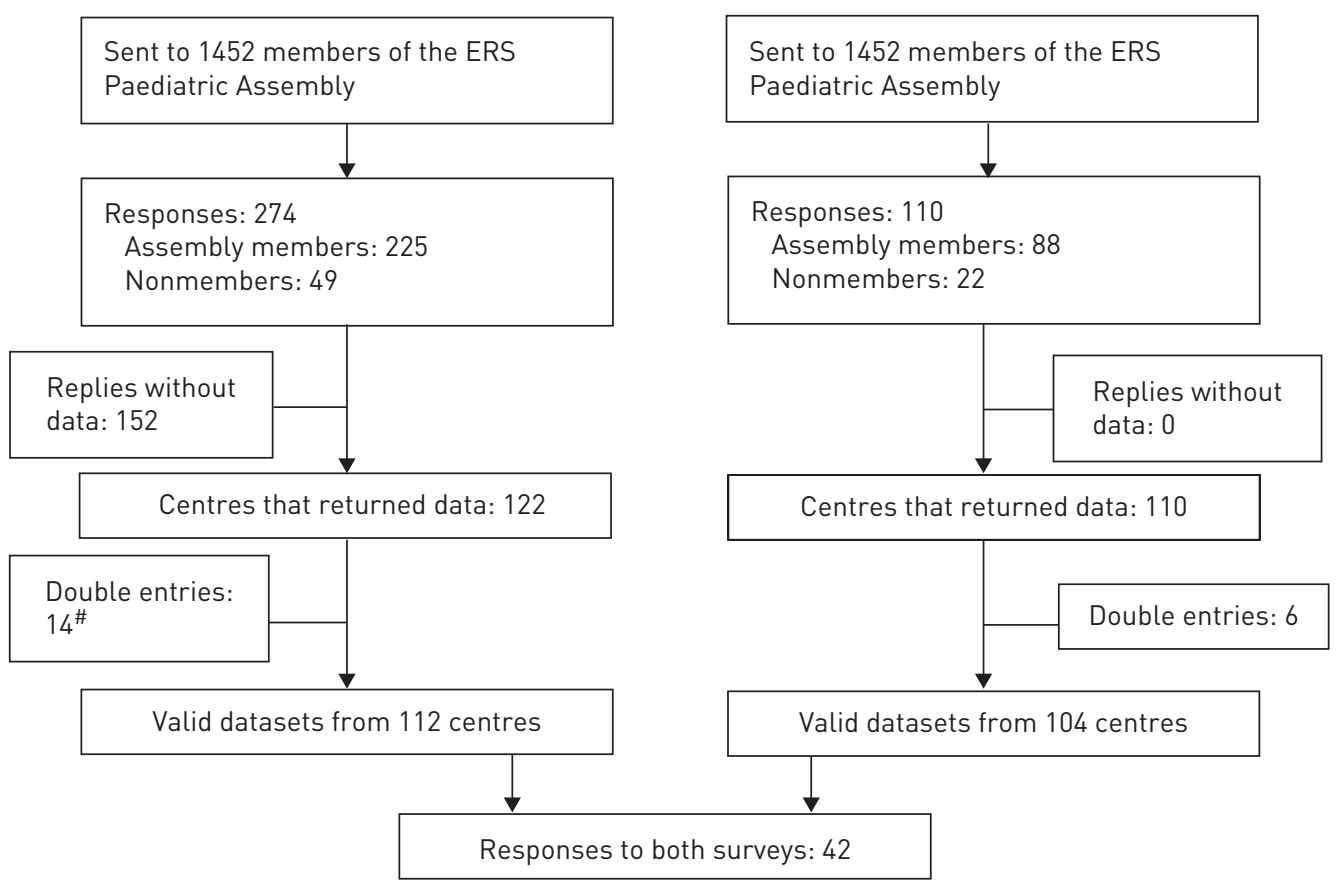

FIGURE 1 Consort diagram. ${ }^{\#}: 4$ of the centres with double entries reported data from different clinics. 
FIGURE 2 Admissions for children with coronavirus disease 2019 (COVID-19) for whom detailed data was provided. Details for children with specific underlying conditions. The bars indicate absolute numbers as presented in the table below. BPD: bronchopulmonary dysplasia; CF: cystic fibrosis; PICU: paediatric intensive care unit; ward: paediatric ward, other: ward not specified.

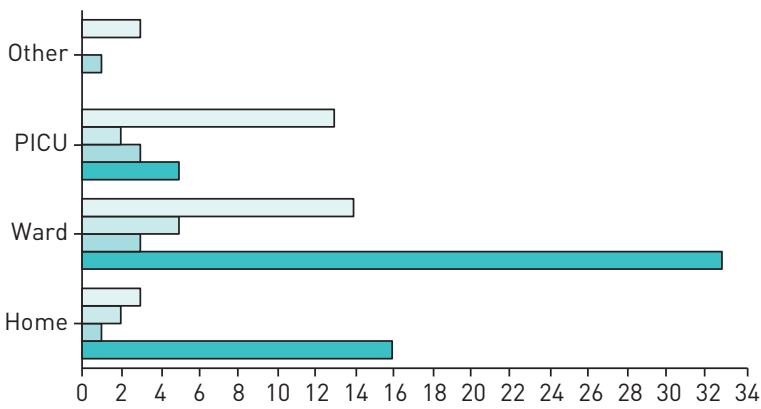

\begin{tabular}{|l|c|c|c|c|}
\cline { 2 - 5 } \multicolumn{1}{c|}{} & Home & Ward & PICU & Other \\
\hline$\square$ Other conditions & 3 & 14 & 13 & 3 \\
\hline$\square$ BPD & 2 & 5 & 2 & 0 \\
\hline$\square$ CF & 1 & 3 & 3 & 1 \\
\hline$\square$ Asthma & 16 & 33 & 5 & 0 \\
\hline
\end{tabular}

2020, but 21 reported new cases between 8 April and 3 May 2020. Follow-up information on cases reported in the first survey was given by 13 respondents, whereas two stated that they did not have follow-up information and one stated that he was not allowed to track the cases.

Data were available for 945 cases of whom 497 (53\%) were boys. Overall, there were 284 (30\%) cases admitted to hospital including 35 (4\%) who were treated in PICU (figure 2). Of the 655 children where data on age was entered, $61 \%$ were older than 6 years and $19 \%$ younger than 1 year (figure 3). Data on underlying conditions was entered for 185 cases. Of those, 63 were asthmatic children, 14 and 9 had a diagnosis of CF and BPD respectively, and 99 had other underlying health conditions.

\section{Asthma}

Details were available for 49 of the 63 cases of asthma, only $10(20 \%)$ of whom presented with an asthma exacerbation. Of the remaining 39 cases, 29 (59\%) presented with upper respiratory tract infection, 16 (33\%) with pneumonia and $12(25 \%)$ with fever only. Some of the children presented with more than one diagnosis, while $14 \%$ had no symptoms of asthma or presented with unclassified symptoms (figure $4 \mathrm{a}$ ). In one example, SARS-CoV-2 was found during the preoperative work-up in an asthmatic child with acute appendicitis. The cases with asthma were on the following Global Initiative for Asthma (GINA) treatment steps: step $1-8$ cases (16\%); step $2-10$ cases (20\%); step 3 - 20 cases (40.8\%); step $4-5$ cases $(10 \%)$; and no child was on step 5 therapy (figure $4 \mathrm{~b}$ ).

Sixteen cases (30\%) were managed at home and 33 were hospitalised to a paediatric ward (61\%), five of whom were admitted to PICU (including one teenager presenting with acute pneumothorax and a history of mild untreated asthma). The median (interquartile range; IQR) length of stay in hospital was 5.5 (8) days. No deaths were reported. There were 19 cases (39\%) who received supplemental oxygen, and four children (8\%) needed invasive ventilation. Nearly two-thirds were treated with antibiotics, and the use of azithromycin was reported in 30 of the asthmatic children. Experimental COVID-19 treatments were used in three of the children. At the time of the second survey, none of the children previously reported to have been managed at home was admitted, and all admitted children had been discharged.

FIGURE 3 Age distribution of children with reported coronavirus disease 2019 (COVID-19) infection. Bars indicate absolute numbers of children with COVID-19.

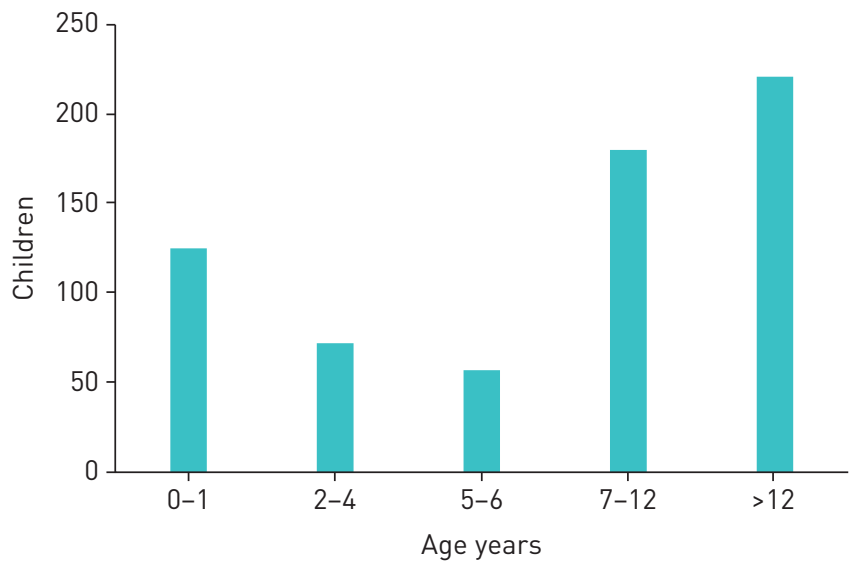



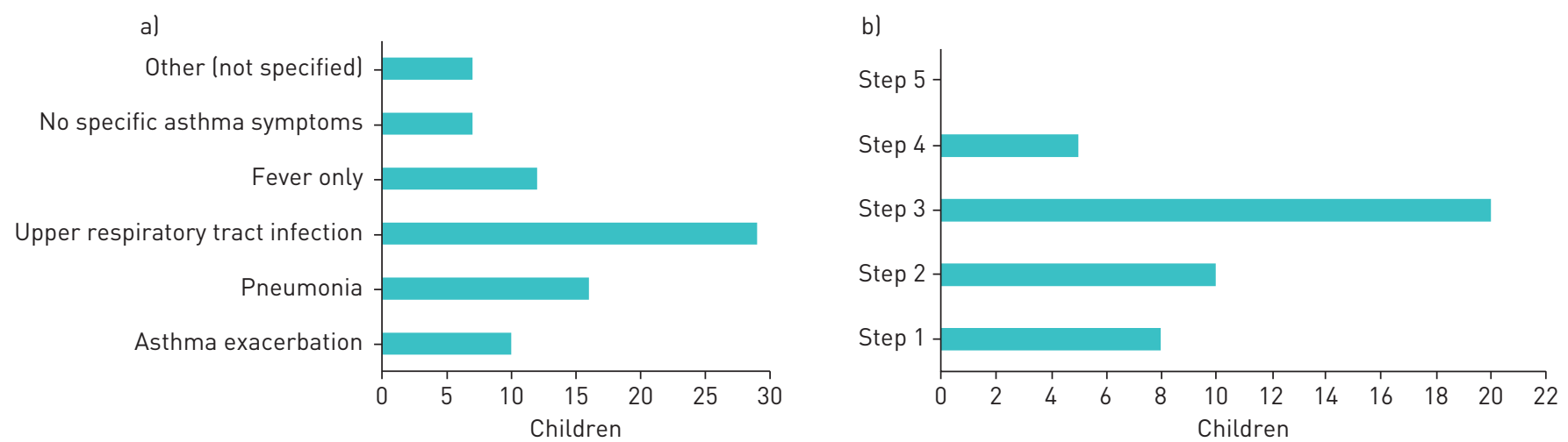

FIGURE 4 a) Leading clinical presentation and symptoms of children with asthma and coronavirus disease 2019 (COVID-19). b) Distribution of children with asthma and COVID-19 in relation to their basic asthma therapy according to Global Initiative for Asthma (GINA) steps. Bars indicate absolute numbers.

\section{Cystic fibrosis}

Details were available for 13 of the 14 cases with CF, four of whom had a pulmonary exacerbation. Additionally, five presented with upper airway tract infection, two with pneumonia and one with fever only. Seven were admitted to hospital, three to PICU and four to a paediatric ward. The median (IQR) length of stay was 3 (15) days. Ten of the 14 children received no therapy, while one needed invasive ventilation and two supplemental oxygen. In three children, the use of antibiotics was reported, and three others received azithromycin as COVID-19 therapy and one hydroxychloroquine. No deaths were reported.

\section{Bronchopulmonary dysplasia}

Information was available for nine cases with BPD of whom there were three in each of the mild, moderate and severe categories. The presenting clinical picture was reported only in three cases and included upper respiratory tract infection, pneumonia and fever. Two were managed at home, five were in a paediatric ward and two admitted to PICU. The median (IQR) length of stay was 7.5 (10) days. One child had never been discharged home. In contrast to the asthmatic children and the CF patients, all infants with BPD received treatment: oxygen use was reported in three children and noninvasive ventilation in four infants, while two received antibiotic treatment and two azithromycin. No deaths were reported.

\section{Other underlying conditions}

There were 33 cases with other underlying conditions (summarised in table 1). Figure 5 shows the clinical presentations. All but three children were admitted to hospital, with 14 being admitted to a paediatric ward and 13 to a PICU, including seven children with an underlying respiratory condition (non-CF bronchiectasis (1), laryngomalacia (1), childhood interstitial lung disease (chILD) (2), pulmonary hypertension (1) and chronic respiratory failure (2)). Three children were admitted to a nonspecified ward. The median (IQR) length of stay was 11 (8) days, with three ongoing hospitalisations at the time of the second survey. Twenty cases needed oxygen, and 11 needed some sort of additional ventilatory support (3 high-flow nasal cannula, 2 NIV and 6 invasive ventilation). Antibiotics were given to 21 children, with azithromycin given to 16 of the 33 children. Experimental COVID-19 treatments were used in six of the children. One infant suffering from chILD was treated with lopinavir/ritonavir, subcutaneous interferon (IFN) $\beta 1 \mathrm{~b}$, remdesivir and hydroxychloroquine, with a hospital stay of 24 days, 13 days of which were in a PICU. One child with congenital diaphragmatic hernia and pulmonary hypertension was admitted to a PICU for 10 days. One death due to acute respiratory distress syndrome was reported in a 2-year-old infant.

\section{Discussion}

This survey was designed to capture the burden of hospitalisations due to COVID-19 among children with pre-existing respiratory conditions such as asthma, CF or BPD. Of the almost 1000 SARS-CoV-2-infected children in this survey, $<10 \%$ of children had underlying respiratory diseases and only very few were admitted to the hospital or to the PICU. A sizeable minority of children with conditions other than asthma and CF with COVID-19 did require invasive or noninvasive ventilation indicating that these subgroups might be susceptible to severe SARS-CoV-2 infection. A recent systematic literature review 


\begin{tabular}{|c|c|c|c|}
\hline Condition & $\mathbf{n}$ & Condition & $\mathbf{n}$ \\
\hline Respiratory conditions & & Underlying neurological conditions & \\
\hline Non-CF bronchiectasis & 10 & Guillain-Barre syndrome & 2 \\
\hline Laryngomalacia & 1 & Mental retardation & 2 \\
\hline Congenital bronchial stenosis & 1 & Rett syndrome & 1 \\
\hline Chronic aspiration pneumonia & 1 & Epilepsy & 2 \\
\hline Pulmonary hypertension & 3 & Hypoxic encephalopathy & 2 \\
\hline Bronchiolitis obliterans & 1 & Ataxia telangiectasia & 1 \\
\hline Interstitial lung disease & 2 & Autism & 1 \\
\hline Surfactant deficit syndrome & 1 & Neuromuscular disease & 1 \\
\hline Tracheostomy & 2 & & \\
\hline Severe bronchomalacia & 1 & Renal conditions & \\
\hline \multirow[t]{2}{*}{ Wheezing } & 1 & Renal transplant & 1 \\
\hline & & Chronic renal insufficiency & 2 \\
\hline Cardiac conditions & & Polycystic renal disease & 1 \\
\hline Congenital heart disease & 6 & Other & 1 \\
\hline \multirow{2}{*}{ Supraventricular arrhythmia } & 1 & & \\
\hline & & Miscellaneous & \\
\hline Oncological/haematological conditions & & Charge syndrome & 1 \\
\hline Unclassified oncological condition & 3 & Down syndrome & 4 \\
\hline Neuroblastoma & 2 & Malformative syndrome & 1 \\
\hline Medulloblastoma & 1 & Crohn disease & 1 \\
\hline Malignancy & 1 & Exostoses & 1 \\
\hline Leukaemia & 3 & Mild malnutrition & 1 \\
\hline Thrombocytosis & 1 & Pierre Robin sequence & 1 \\
\hline \multirow[t]{4}{*}{ SCID } & 1 & Prader-Willi syndrome & 1 \\
\hline & & Liver transplantation & 1 \\
\hline & & Portal hypertension & 1 \\
\hline & & Diabetes mellitus & 1 \\
\hline
\end{tabular}

evaluated reported paediatric cases of COVID-19 from China and Singapore published between December 2019 and March 2020 including a total of 1065 children with SARS-CV-2 infection. Of these children, 444 were younger than 10 years of age at the time of infection. Symptoms were generally mild and included mainly respiratory complaints including dry cough and systemic symptoms such as fever and fatigue. Some children presented with gastrointestinal symptoms. Only one case with severe lower respiratory tract infection necessitating intensive care and no deaths were presented. No information on respiratory comorbidities was given in the 18 reports included [8].

In our study, most data were on SARS-CoV-2-infected children with asthma. Although respiratory viruses such as rhinoviruses are well-known triggers for asthma exacerbations, only a small minority of SARS-CoV-2-infected children presented with an asthma exacerbation. This is in line with data in adults, in whom asthma has not been shown to be a significant risk factor for severe COVID-19 [9]. In the USA, 67 children with COVID-19 were reported including 11 children with asthma: 33 (8 asthmatic) were admitted to the ward and 13 (3 asthmatic) were admitted to the PICU between March 15 and April 14, 2020. The authors reported that obesity and asthma were highly prevalent, but these comorbidities were not significantly associated with PICU admission [10]. Similarly, during the SARS-CoV (2002) and Middle East respiratory syndrome (MERS) (2012) epidemics there was no increased rate of asthma exacerbations, and children appeared less susceptible to infection than adults $[11,12]$.

It has been speculated that decreased angiotensin converting enzyme 2 (ACE2) expression in allergic asthmatic children may explain the low rate of asthma exacerbations. Sars-CoV-2 uses ACE2 as its cellular receptor, expressed on ciliated bronchial cells and Type I and II alveolar cells. In children and adults with allergic asthma, ACE2 expression is reduced with lowest expression in patients with the highest levels of allergic sensitisation $[13,14]$.

In a recent report from Italy only two children with allergies and one child with asthma out of a total of 40 children with SARS-CoV-2 infection were described, although the prevalence of allergies and asthma are $30 \%$ and $11.6 \%$ respectively. Interestingly, all three children had lower total blood eosinophil counts than a control group of 120 allergic children from the same region [15]. Eosinopenia, most probably caused by $\mathrm{CD}^{+}$T-cell and eosinophil depletion by SARS-CoV-2, has been shown to be associated with 
FIGURE 5 Leading clinical presentation and symptoms of children with other underlying conditions and coronavirus disease 2019 (COVID-19). Bars indicate absolute numbers.

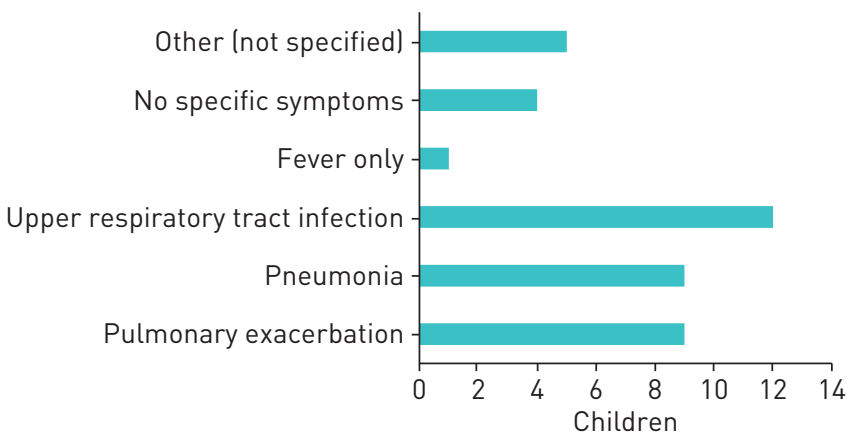

more severe outcome and higher mortality in adult COVID-19 patients [16]. As eosinophils may play an important role in the immune response to viral respiratory tract infections by reducing the viral load, patients with elevated eosinophils, hence allergic subject, may be less affected by COVID-19.

Another contributing factor to the low number of asthma exacerbations is the likely lack of exposure to viruses known to trigger asthma attacks, in particular rhinoviruses, as a consequence of social isolation, the closure of schools, step-up of public hygiene measures and the wearing of facemasks [11].

Remarkably, 70\% of all asthmatic children with COVID-19 in our survey were admitted to hospital. Only half of them received supplemental oxygen, which may suggest that children were admitted for safety reasons and uncertainty about the course of COVID in children. Five children with asthma (out of 49) were admitted to the PICU and four needed invasive ventilation. In general, there is a peak in PICU admissions for asthma in March to April when the tree pollen season starts, and we speculate that some admissions may have been caused by pollen exposure and coincidental SARS-CoV-2 infection.

In our survey only 14 children with CF and COVID-19 were reported until May 3, 2020. This number is very comparable to data from the European Cystic Fibrosis Society database that as of June 8, 2020 included 16 children with CF (www.ecfs.eu/covid-cf-project-europe). Also, other data suggest that there were few infected CF patients, who were mainly adults, and no deaths occurred. Again, the majority of children did not receive therapy including supplemental oxygen, suggesting a mild course and a low threshold for admission. However, one child with CF needed mechanical ventilation, but we do not have any pre-COVID data on this patient. Also for CF, the strict hygiene measures that were in place anyway and also strict isolation early in the pandemic may have protected patients against severe COVID-19.

For all other underlying respiratory diseases BPD was reported in nine and non-CF bronchiectasis in 10 children with COVID-19; other underlying diseases were very heterogeneous in nature.

Although the vast majority of children had mild symptoms, 10 children in our cohort were treated with experimental COVID-19 treatments despite the lack of evidence for such treatments; however, we do not know the clinical condition of these children and the reasons why this treatment was started.

In our survey 35 children were admitted to a PICU, and 17 had an underlying respiratory disease. This figure is much higher than that from a study in the USA, where two children out of 48 admitted to the PICU had a chronic lung disease [17]. This may be explained by the fact that our survey contacted paediatric pulmonologists rather than intensive care paediatricians.

Our study has several strengths. To date this is the largest data set of children with COVID-19 with underlying respiratory conditions, where outcomes of hospitalisation are described. We accessed well-established expert's networks within the ERS, the biggest respiratory society worldwide. This made it possible to obtain timely information on the effects of this new virus in a vulnerable paediatric population that instantly could be shared with colleagues all over the world. A broad range of sites contributed data, which make our results generalisable. Our results could be used to reassure patients and carers of children with asthma and CF that SARS-CoV-2 acquisition is not usually associated with serious infection (i.e. requiring ventilatory support) and the outcome is almost invariably good.

Our study has several limitations. We choose to use a simple survey, without too much detail, but retrieving essential information as soon as possible. The response rate of the survey was around $20 \%$, therefore we will have missed paediatric cases. However, it was not our aim to include all European cases, but rather to get quick information on the risks of children with respiratory conditions [18]. The majority of participants were not able to contribute data, and although at least one centre in most countries in Europe did contribute data, these findings may not be generalisable to every region. Second, we do not have extensive clinical follow-up data on the children included in the survey, and we will have missed most 
asymptomatic or mildly symptomatic paediatric patients as the data presented here are hospital based. However, we believe that even when parents were reluctant to bring their child to hospital during the SARS-CoV-2 pandemic, children requiring supplemental oxygen and ventilatory support will have been brought to hospital and captured in our survey. Third, detecting the virus in children is challenging (especially in younger children) and false-negative cases with pre-existing respiratory conditions and admitted to hospital will have been excluded. Fourth, some important hotspots of the COVID-19 pandemic were not included in the survey (e.g. USA, Brazil and China). Finally, policies to handle SARS-CoV-2 in countries were numerous and differed among countries. Timing and severity of segregation strategies, lockdown protocols and hygiene measures are too complex for description in this article; however, in countries where diagnosis was shifted to structures outside the hospital setting, detection of paediatric cases was even less likely.

In summary, we report a survey of outcomes after hospitalisation with COVID-19 in children and young people with pre-existing respiratory illnesses such as asthma and CF. The low numbers of children with these conditions admitted to hospital suggest that these children are not at increased risk for severe COVID-19, and these emerging findings may be useful for governments planning for provision of care over the coming months and years. Still, a sizeable minority of children with BPD and respiratory diseases other than asthma might be at increased risk and may benefit from being shielded.

Acknowledgements: We thank all participating centres for providing data during this hectic and uncertain time, and the European Respiratory Society for the technical support.

Conflict of interest: A. Moeller has nothing to disclose. L. Thanikkel has nothing to disclose. L. Duijts has nothing to disclose. E.A. Gaillard has nothing to disclose. L. Garcia-Marcos has nothing to disclose. A. Kantar has nothing to disclose. N. Tabin is an employee of the European Respiratory Society. S. Turner has nothing to disclose A. Zacharasiewicz reports financial compensation for participation in advisory boards from Vertex, Novartis, Chiesi, Gilead and AOP, and lecture fees from Hagleitner Hygiene, AstraZeneca, and Heine und Löwenstein. M.W.H. Pijnenburg has nothing to disclose.

\section{References}

1 World Health Organization. Coronavirus disease 2019 (COVID-19) Situation Report - 79. www.who.int/docs/ default-source/coronaviruse/situation-reports/20200408-sitrep-79-covid-19.pdf?sfvrsn=4796b143_6 Date last accessed: 7 July 2020. Date last updated: 8 April 2020.

2 Livingston E, Bucher K. Coronavirus disease 2019 (COVID-19) in Italy. JAMA 2020; 323: 1335.

3 Zhang Y. The Novel Coronavirus Pneumonia Emergency Response Epidemiology Team. The epidemiological characteristics of an outbreak of 2019 novel coronavirus diseases (COVID-19) in China. Zhonghua Liu Xing Bing Xue Za Zhi 2020; 10: 145-151.

4 Bialek S, Boundy E, Bowen V, et al. Severe outcomes among patients with coronavirus disease 2019 (COVID-19) United States, February 12-March 16, 2020. MMWR Morb Mortal Wkly Rep 2020; 69: 343-346.

5 Dong Y, Mo X, Hu Y, et al. Epidemiology of COVID-19 among children in China. Pediatrics 2020; 145: e20200702.

6 Verdoni L, Mazza A, Gervasoni A. An outbreak of severe Kawasaki-like disease at the Italian epicentre of the SARS-CoV-2 epidemic: an observational cohort study. Lancet 2020; 395: 1771-1778.

7 Qifang B, Yongsheng W, Shujiang M, et al. Epidemiology and transmission of COVID-19 in Shenzhen China: analysis of 391 cases and 1286 of their close contacts. MedRxiv 2020; preprint [https://10.1101/2020.03.03.20028423].

8 Castagnoli R, Votto M, Licari A, et al. Severe acute respiratory syndrome coronavirus 2 (SARS-CoV-2) infection in children and adolescents: a systematic review. JAMA Pediatr 2020; 174: 882-889.

9 Li X, Xu S, Yu M, et al. Risk factors for severity and mortality in adult COVID-19 inpatients in Wuhan. J Allergy Clin Immunol 2020; 146: 110-118.

10 Chao JY, Derespina KR, Herold BC, et al. Clinical characteristics and outcomes of hospitalized and critically ill children and adolescents with coronavirus disease 2019 (COVID-19) at a tertiary care medical center in New York City. J Pediatr 2020; 223: 14-19.

11 Van Bever HP, Chng SY, Goh DY. Childhood severe acute respiratory syndrome, coronavirus infections and asthma. Pediatr Allergy Immunol 2004; 15: 206-209.

12 Bartenfeld M, Griese S, Uyeki T, et al. Middle East respiratory syndrome coronavirus and children. Clin Pediatr (Phila) 2017; 56: 187-189.

13 Jackson DJ, Busse WW, Bacharier LB, et al. Association of respiratory allergy, asthma, and expression of the SARS-CoV-2 receptor ACE2. J Allergy Clin Immunol 2020; 146: 203-206.

14 Kimura H, Francisco D, Conway M, et al. Type 2 inflammation modulates ACE2 and TMPRSS2 in airway epithelial cells. J Allergy Clin Immunol 2020; 146: 80-88.

15 Licari A, Votto M, Brambilla I, et al. Allergy and asthma in children and adolescents during the COVID outbreak: what we know and how we could prevent allergy and asthma flares. Allergy 2020; 75: 2402-2405.

16 Li Q, Ding X, Xia G, et al. Eosinopenia and elevated C-reactive protein facilitate triage of COVID-19 patients in fever clinic: a retrospective case-control study. EClinicalMedicine 2020; 23: 100375.

17 Shekerdemian LS, Mahmood NR, Wolfe KK, et al. Characteristics and outcomes of children with coronavirus disease 2019 (COVID-19) infection admitted to US and Canadian pediatric intensive care units. JAMA Pediatr 2020; 174: 868-873.

18 Safdar N, Abbo LM, Knobloch MJ, et al. Research methods in healthcare epidemiology: survey and qualitative research. Infect Control Hosp Epidemiol 2016; 37: 1272-1277. 